\title{
Análisis de la Distribución del tiempo laboral para la espe- cialidad de medicina interna en los hospitales de la Caja Costarricense de Seguro Social, Costa Rica, 2014
}
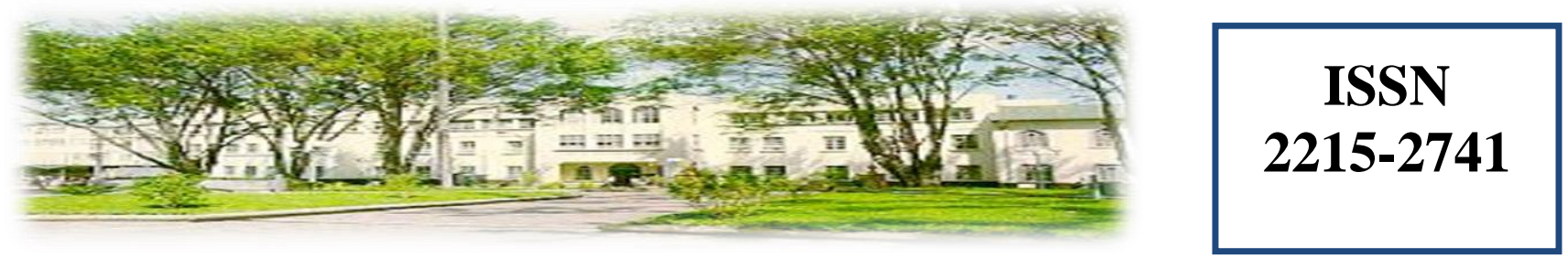

Hospital San Juan de Dios, San José, Costa Rica. Fundado en 1845

Recibido:

$6 / 12 / 16$

Aceptado:

$9 / 12 / 2016$

Angie Cervantes Rodríguez ${ }^{1}$

Daniel Murillo Castro ${ }^{2}$

\begin{abstract}
${ }^{1}$ Microbióloga. Máster en Administración de Servicios de Salud. Dirección Regional de Servicios de Salud Central Sur. C.C.S.S. San José Costa Rica.

${ }^{2}$ Especialista en Medicina Interna. Máster en Administración de Servicios de Salud. Hospital San Juan de Dios. C.C.S.S. San José. Costa Rica.
\end{abstract}

\section{RESUMEN}

La distribución del tiempo laboral y las actividades realizadas está relacionada con la calidad y la productividad. Se evalúa el tiempo recomendable de consulta y distribución del tiempo de los médicos internistas con el fin de evitar la sobrecarga laboral y mejorar la atención.

Se aplicó una encuesta a 42 médicos internistas de hospitales nacionales, periféricos y regionales de la CCSS. Se encontró una diferencia estadísticamente significativa $(\mathrm{p}=0,03)$ entre el tiempo de atención de consulta externa entre hospitales periféricos y nacionales y el tiempo dedicado a la academia de los hospitales periféricos y los nacionales ( $p<0,01)$. El tiempo de consulta varía significativamente según el tipo de hospital, el tipo de consulta (primera vez, subsecuente o interconsulta) y los años de experiencia laboral del médico internista. Las actividades dentro de la consulta presentan un alto nivel de cumplimiento pero el tiempo percibido por los médicos para realizar la consulta en todos los casos difiere por más de 20 minutos del deseado.

Se debe realizar una revisión a los tiempos de consulta de los médicos internistas y adecuarlos a las necesidades de la especialidad.

\section{PALABRAS CLAVE}

Medicina interna. Administración. Tiempo. Hospitalización. Consulta Externa. Interconsulta. Consulta subsecuente.

\section{ABSTRACT}

The distribution of working time and activities are related to quality and productivity. The recommended time of consultation and distribution of the time of the internists are evaluated, in order to avoid work overload and improve health care.

A survey was applied to 42 internists from national, peripheral and regional hospitals of the CCSS. A statistically significant difference $(p=0,03)$ was found between outpatient care time of peripheral and national hospitals and the time spent in the academy of peripheral and national hospitals ( $p<0.01$ ). Consultation time varies significantly according to the type of hospital, the type of consultation (first time, or subsequent consultation) and years of work experience of the internist. The activities within the consultation have a high level of compliance but the time perceived by the doctors to perform the consultation in all cases differs by more than 20 minutes from the desired one. 
A review of the consultation times of the internists must be made and adapted to the needs of the specialty.

\section{KEY WORDS}

Internal Medicine. Administration. Working time. Hospitalization. External Consultation. Interconsulting. Subsequent consultation.

\section{INTRODUCCIÓN}

Los servicios de salud han de perseguir la calidad de la atención disminuyendo la brecha entre las necesidades de los usuarios y la oferta de servicios sin que esto se traduzca en sobrecarga laboral para los profesionales. Los médicos internistas no tienen una distribución del tiempo de las actividades y los procesos de su jornada laboral. La OPS recomienda la atención de 4 pacientes por hora (9) pero no se refiere a qué tipo de médicos aplica. La Ley General de Salud de Costa Rica indica que un hospital debe tener 1 médico por cada 12 pacientes internados (6) y el Colegio de Médicos dispone que un médico especialista debería atender 4 pacientes subsecuentes por hora y 2 por primera vez (2), más no establece diferencias para cada una de las especialidades.

El médico internista entre sus funciones atiende consulta externa por primera vez y subsecuente, interconsultas, hospitalización y realiza actividades académicas y administrativas. Su consulta tiene la particularidad de dar atención a padecimientos crónicos mediante un abordaje integral que demanda una importante inversión de tiempo para garantizar la ética, la seguridad y la comodidad con el paciente.

Se busca determinar la distribución del tiempo de los médicos internistas en sus actividades de la jornada laboral evaluando el cumplimiento de los procesos en cada una de las consultas y si incide en esto el tipo de hospital, el tipo de consulta y la experiencia laboral del médico.

Se desea conocer si el tiempo del que disponen los médicos internistas en la realidad corresponde con el tiempo ideal que desearían para realizar sus labores.
Se realizó un estudio de tiempos y movimientos, descriptivo, observacional y prospectivo con enfoque mixto. Se incluyeron en el estudio médicos especialistas en medicina interna como única especialidad, que laboraran para hospitales de la CCSS, y que realizaran al menos una vez por semana las actividades estudiadas y en disposición de brindar la información.

Se aplicó una encuesta a 42 médicos internistas de 23 hospitales del país. Partiendo de la población total de 80 médicos se obtuvo un $52,5 \%$ como muestra, distribuida en 14 médicos $(33 \%)$ de hospitales periféricos, 15 médicos $(36 \%)$ de hospitales nacionales y 13 médicos $(31 \%)$ de hospitales regionales

Se evaluaron cuatro de las principales actividades de los médicos internistas: consulta externa por primera vez y subsecuente, interconsultas y consulta de hospitalización.

Se evaluó el cumplimiento del proceso de consulta en cada una de estas actividades y los factores que inciden en los tiempos de consulta. Se evaluó si el tiempo percibido de consulta coincidía con el deseado.

Los resultados se analizaron con tablas de distribución de frecuencias para los datos generales y luego para comparaciones con el tiempo de los procesos percibido, deseado y asignado.

Los resultados de las comparaciones de tiempos se sometieron a pruebas de significancia estadística, se utilizaron las pruebas no paramétricas de Kruskal Wallis y la U de Mann Whitney, dado que la muestra era pequeña. En ambos análisis se consideró significativo a un nivel de confianza $<0,05$.

\section{RESULTADOS}

De los médicos encuestados 6 de ellos (14\%) fueron mujeres y los 36 restantes $(86 \%)$ hombres. Con respecto a la experiencia laboral, osciló en un rango de 1 a 36 años, el 70\% de los médicos poseía más de 7 años de experiencia y la media fue de 12.5 años. 
Gráfico 1. Promedio de horas semanales asignadas para los diferentes procesos a los médicos internistas en hospitales de la CCSS, Costa Rica, 2014.



Fuente: Encuesta Análisis de la distribución del tiempo laboral para la especialidad de medicina interna en los hospitales de la Caja Costarricense de Seguro Social, Costa Rica, 2014

Los resultados del uso del tiempo en los procesos evaluados se muestran en los gráficos 1 y 2 . Los dos procesos que demandan más tiempo son la consulta externa y hospitalización. Se encontró una diferencia estadísticamente significativa $(\mathrm{p}=$ 0,03 ) en el tiempo dedicado a la atención de consulta externa entre hospitales periféricos y nacionales. En los hospitales periféricos la atención de la consulta externa demanda a los internistas cerca del $40 \%$ del tiempo semanal (16,2 horas), mientras que en los hospitales nacionales baja a aproximadamente $18 \%$ (7,9 horas).

Gráfico 2. Promedio de horas semanales asignadas para los diferentes procesos a los médicos internistas según tipo de hospital en la CCSS, Costa Rica, 2014.

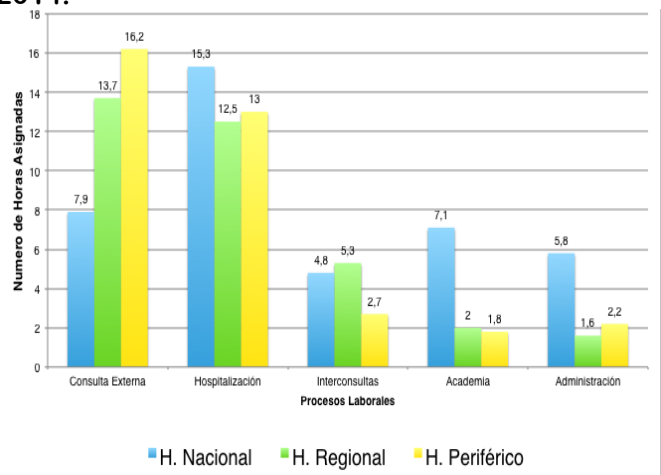

Fuente: Encuesta Análisis de la distribución del tiempo laboral para la especialidad de medicina interna en los hospitales de la Caja Costarricense de Seguro Social, Costa Rica, 2014

En el rubro de horas asignadas para la academia se encontró una diferencia estadísticamente significativa: los hospitales nacionales dedican más horas a la academia que los otros tipos de hospitales $(\mathrm{p}<0,01)$, mostrándose una diferencia neta de 5 horas de más en los hospitales nacionales.

\section{Carga laboral}

Se determinó que en consulta externa por primera vez los médicos atienden de 1 a 5 pacientes por hora siendo la moda 2 y en consulta subsecuente de 2 a 10 pacientes con moda de 4 . El $80 \%$ de los médicos refiere aceptar recargos y en promedio atienden 7,6 como recargo por semana.

Con respecto a la consulta de hospitalización se determinó que los médicos atienden de 0 a 36 camas con un promedio de 11,4 camas por médico y una moda de 6 camas por médico. Si se relaciona el número de camas entre las horas asignadas el promedio es 5,2 pacientes hospitalizados por hora, pero se observa que algunos médicos tienen asignado tiempo pero no camas y otros médicos deben atender hasta 20 pacientes por hora. Los promedios pacientes hospitalizados atendidos por hora fueron de 4,6; 4,5 y 6,6 pacientes hospitalizados nacionales, regionales y periféricos respectivamente, sin diferencias significativas entre ellos.

En promedio los médicos encuestados realizan 10,5 interconsultas semanales, 14 médicos deben atender interconsultas sin tener tiempo asignado para esta labor y de los que tienen asignado la relación es de 3,1 interconsultas por hora.

Cumplimiento de las actividades de consulta Se evaluó el cumplimiento las diferentes actividades en cada tipo de consulta, se detallan los resultados en los siguientes cuadros.

\begin{tabular}{|l|c|}
\hline \multicolumn{2}{|c|}{$\begin{array}{c}\text { Cuadro 1: Porcentajes de Cumplimiento de Actividades } \\
\text { en el Proceso de Consulta Externa por Primera Vez por } \\
\text { Médicos Internistas en Hospitales de la CCSS Costa Rica, } \\
\text { Actividades }\end{array}$} \\
\hline \multicolumn{2}{|c|}{ Porcentaje } \\
\hline Recibir al paciente & $\mathbf{8 1 , 7}$ \\
\hline Realizar historia clínica completa & 79,3 \\
\hline Lavarse las manos (primera vez) & 67,1 \\
\hline Realizar examen físico completo & 73,2 \\
\hline Lavarse las manos (segunda vez) & 64 \\
\hline Revisión de expediente & 79,9 \\
\hline $\begin{array}{l}\text { Elaboración del diagnóstico y expli- } \\
\text { cación de este al paciente }\end{array}$ & 92,7 \\
\hline $\begin{array}{l}\text { Explicación del plan de estudio o tra- } \\
\text { tamientos al paciente }\end{array}$ & 89,6 \\
\hline $\begin{array}{l}\text { Dar recomendaciones de salud al } \\
\text { paciente }\end{array}$ & 87,2 \\
\hline Actividades administrativas & 94,5 \\
\hline
\end{tabular}


Fuente: Encuesta Análisis de la distribución del tiempo laboral para la especialidad de medicina interna en los hospitales de la Caja Costarricense de Seguro Social, Costa Rica, 2014

Con respecto al proceso de la consulta externa por primera vez se encontró un $82 \%$ de cumplimiento general que es similar a la consulta subsecuente con $83,8 \%$ y en los siguientes cuadros se pueden observar las diferentes actividades. El valor de cumplimiento más bajo fue en el lavado de manos (lo cual es una actividad esencial en la prevención de enfermedades infectocontagiosas) y el más alto fue en las actividades administrativas.

\begin{tabular}{|c|c|}
\hline \multicolumn{2}{|c|}{$\begin{array}{l}\text { Cuadro 2: Porcentajes de Cumplimiento de Actividades } \\
\text { en el Proceso de Consulta Externa por Subsecuente por } \\
\text { Médicos Internistas en Hospitales de la CCSS Costa Rica, } \\
2014\end{array}$} \\
\hline Actividades & Porcentaje \\
\hline Recibir al paciente & 83,1 \\
\hline Realizar historia clínica & 84,4 \\
\hline Lavarse las manos (primera vez) & 66,3 \\
\hline Realizar examen físico & 80 \\
\hline Lavarse las manos (segunda vez) & 62,5 \\
\hline Revisión de expediente & 86,9 \\
\hline $\begin{array}{l}\text { Confirmación del diagnóstico y ex- } \\
\text { plicación de este al paciente }\end{array}$ & 90 \\
\hline Despedida del paciente & 92.7 \\
\hline
\end{tabular}

Fuente: Encuesta Análisis de la distribución del tiempo laboral para la especialidad de medicina interna en los hospitales de la Caja Costarricense de Seguro Social, Costa Rica, 2014

En los procesos de hospitalización los cumplimientos fueron de $86,8 \%$ y 85,2 \% de forma general para consulta hospitalaria e interconsulta respectivamente (Cuadro 3 y 4), esto es levemente mejor que en consulta externa y de nuevo la actividad de menor cumplimiento fue el lavado de manos.

\begin{tabular}{|c|c|}
\hline \multicolumn{2}{|c|}{$\begin{array}{c}\text { Cuadro 3: Porcentajes de Cumplimiento de Actividades } \\
\text { en el Proceso de Consulta de Hospitalización por Médi- } \\
\text { cos Internistas en Hospitales de la CCSS Costa Rica, } \\
2014\end{array}$} \\
\hline Actividades & Porcentaje \\
\hline Desplazarse hasta el salón & 99,4 \\
\hline Buscar el expediente del paciente & 92,1 \\
\hline $\begin{array}{c}\text { Saludar y presentarse con el pa- } \\
\text { ciente }\end{array}$ & 90,9 \\
\hline Realizar historia clínica & 81,7 \\
\hline Lavarse las manos (primera vez) & 78 \\
\hline Realizar examen físico & 79,9 \\
\hline Lavarse las manos (segunda vez) & 72,6 \\
\hline Revisión de expediente & 94,5 \\
\hline $\begin{array}{c}\text { Revisión de hoja de medicamentos } \\
\text { (Kardex) }\end{array}$ & 90,9 \\
\hline $\begin{array}{l}\text { Explicación del diagnóstico, trata- } \\
\text { miento y evolución al paciente }\end{array}$ & 92,7 \\
\hline Actividades administrativas & 82,3 \\
\hline
\end{tabular}

La actividad de mayor cumplimiento en hospitalización e interconsulta es la revisión del expediente luego de las de desplazarse al salón o buscar el expediente. Llama la atención que en la interconsulta la actividad menos realizada es la comunicación con el médico interconsultante.

\begin{tabular}{|c|c|}
\hline \multicolumn{2}{|c|}{$\begin{array}{l}\text { Cuadro 4: Porcentajes de Cumplimiento de Actividades } \\
\text { en el Proceso de Interconsulta por Médicos Internistas } \\
\text { en Hospitales de la CCSS Costa Rica, } 2014\end{array}$} \\
\hline Actividades & Porcentaje \\
\hline Ir por la interconsulta & 83,6 \\
\hline Desplazarse hasta el salón & 98,0 \\
\hline Buscar el expediente del paciente & 96,7 \\
\hline $\begin{array}{l}\text { Saludar y presentarse con el pa- } \\
\text { ciente }\end{array}$ & 96,7 \\
\hline Realizar historia clínica completa & 86,8 \\
\hline Lavarse las manos (primera vez) & 77,0 \\
\hline Realizar examen físico & 80,3 \\
\hline Lavarse las manos (segunda vez) & 69,7 \\
\hline Revisión de expediente & 95,4 \\
\hline Revisión de hoja de medicamentos & 91,4 \\
\hline $\begin{array}{l}\text { Explicación del diagnóstico, trata- } \\
\text { miento y evolución al paciente }\end{array}$ & 88,8 \\
\hline Actividades administrativas & 86,2 \\
\hline $\begin{array}{l}\text { Realizar las recomendaciones de la } \\
\text { interconsulta }\end{array}$ & 90,1 \\
\hline $\begin{array}{l}\text { Comunicarse con médico intercon- } \\
\text { sultante }\end{array}$ & 51,3 \\
\hline
\end{tabular}

Tiempo percibido vs tiempo deseado

De la evaluación del tiempo percibido de cada consulta por los médicos internistas y lo deseado se desprende el Cuadro 5.

\begin{tabular}{|c|c|c|c|}
\hline \multicolumn{3}{|c|}{$\begin{array}{c}\text { Cuadro 5: Promedio de los Tiempos Percibidos y Desea- } \\
\text { dos y la Diferencia entre estos en los diferentes proce- } \\
\text { sos de los Médicos Internistas de Hospitales de la CCSS } \\
\text { Costa Rica, 2014 }\end{array}$} \\
\hline Procesos & $\begin{array}{c}\text { Perci- } \\
\text { bido }\end{array}$ & Deseado & Diferencia \\
\hline $\begin{array}{c}\text { Tiempo Con- } \\
\text { sulta por pri- } \\
\text { mera vez }\end{array}$ & 38,5 & 74,6 & 36,1 \\
\hline $\begin{array}{c}\text { Tiempo Con- } \\
\text { sulta Subse- } \\
\text { cuente }\end{array}$ & 29,5 & 51,8 & 22,3 \\
\hline $\begin{array}{c}\text { Tiempo Con- } \\
\text { sulta de Hos- } \\
\text { pitalización }\end{array}$ & 41,7 & 69,4 & 27,7 \\
\hline $\begin{array}{c}\text { Tiempo para } \\
\text { Interconsulta }\end{array}$ & 49,6 & 72,5 & 22,9 \\
\hline \multicolumn{4}{|c|}{ Tiempo en minutos } \\
\hline
\end{tabular}

Fuente: Encuesta Análisis de la distribución del tiempo laboral para la especialidad de medicina interna en los hospitales de la Caja Costarricense de Seguro Social, Costa Rica, 2014 
De forma general se observa una diferencia de más de 20 minutos en los 4 procesos estudiados, de forma general los médicos desean mayor tiempo para realizar estos procesos. En donde se observó mayor diferencia fue en la consulta por primera vez, que es cuando se realiza la historia clínica completa y cuando se conoce al paciente. Se estudiaron estas diferencias con relación a los años de experiencia de los médicos y se obtuvo que en el proceso de consulta externa por primera vez el tiempo percibido actual es significativamente menor para los médicos con menos años de experiencia $(p=0,007)$. Es de resaltar que no se encontró diferencia significativa entre el tiempo deseado de los diferentes hospitales, lo cual indica que todos los médicos desean más tiempo y están inconformes con el tiempo actual.

\section{DISCUSIÓN}

De las diferencias significativas del estudio en cuanto a la distribución del tiempo para los diferentes procesos son significativos el mayor tiempo dedicado a la consulta externa en los hospitales periféricos con respecto a los nacionales y lo contrario con respecto al tiempo académico. La utilización del tiempo de academia en los hospitales nacionales está relacionado con la formación de estudiantes en estos hospitales y la dedicación a tareas como sesiones clínicas. La calidad de la atención está relacionada con la formación que tengan los profesionales y en este caso en los hospitales nacionales existe una mayor oportunidad de la formación continua.

Con respecto a la carga laboral, fluctúa la cantidad de pacientes atendidos en cada uno de los procesos, pero en todos ellos se llegan a atender en algunos hospitales más de los pacientes recomendados por el Colegio de Médicos (2). Cada proceso tiene sus particularidades, pero de manera general cuando se atienden más de los pacientes recomendados por hora, las consultas son rápidas y el médico toma decisiones menos efectivas, incurre en solicitud de estudios que ya tiene o no necesita y uso de medicamentos que el paciente no requiere, también se pueden dar errores diagnósticos y menos altas de la consulta.

En el caso de hospitalización las actividades administrativas disminuyen y lo que se puede inferir es que existen otros médicos en salón encargados de estas. En la mayoría de los casos los médicos cuentan con mayor tiempo para la atención intrahospitalaria. La interconsulta se podría ver beneficiada con el mejoramiento de disponibilidad de la interconsulta y el expediente y la comunicación con el médico interconsultante.

Con respecto a los tiempos percibidos y deseados de forma general se observa una diferencia de más de 20 minutos en los 4 procesos estudiados, los médicos desean más tiempo para realizarlos. Esto contradice la aceptación de recargos, pero puede responder a que son favores a conocidos o compañeros de trabajo, pacientes con una necesidad puntual de revisar un resultado específico o pacientes graves. En donde se observó mayor diferencia fue en la consulta por primera vez, que es cuando se realiza la historia clínica completa y cuando se conoce al paciente, esto es importante para todas las especialidades pero particularmente para la especialidad de medicina interna que es una especialidad integral, por lo que se propone aumentar la consulta de primera vez a 40 minutos en lugar de 30 y la subsecuente a 20 minutos en lugar de 15, 30 minutos para cada paciente hospitalizado y para cada interconsulta, esto presupuesta tiempo para posibles procedimientos a los pacientes internados y eventuales emergencias. Esto debe ir concomitante a incentivar el cumplimiento de las actividades completas y mejorar la calidad de atención.

Nuestra propuesta para los jefes de servicio es realizar el cronograma tomando en cuenta al trabajador, en primer lugar decidir el tiempo de hospitalización y en el restante organizar los demás procesos, sin excluir academia y tiempo administrativo. Esto a la luz de la dinámica que comprende la medicina interna como una especialidad que ha de atender una prevalencia en aumento de las enfermedades crónicas y un aumento en la expectativa de vida de los pacientes.

\section{CONCLUSIONES}

Las actividades a las cuales dedica el tiempo un médico internista según el tipo de hospital, varían significativamente en algunos casos. La mayor inversión de horas de la jornada laboral se hace en la atención de consulta eterna y hospitalización sin distinción del tipo de hospital. Se dan diferencias significativas entre el tiempo que es dedicado a la atención de consulta externa en los hospitales periféricos que es mayor que en los hospitales nacionales y en el tiempo dedicado a la academia que es significativamente menor en los hospitales periféricos.

Los pacientes que son atendidos por primera vez requieren hasta el doble de tiempo de atención que en consulta subsecuente. No se puede estandarizar 
un tiempo de consulta externa sino que se debe tomar en cuenta si es primera vez o subsecuente. Se encontraron diferencias significativas en la cantidad de pacientes que atiende cada médico en los diferentes centros en un rango que va de 0 a 20 camas. Esto afecta de manera directa la calidad de la atención y se podría presumir una sobrecarga de trabajo para los médicos internistas que atienden más que el promedio de 6 camas.

Las interconsultas no están consideradas dentro de las actividades del internista por lo que resultan en sobrecarga de trabajo. Tanto en consulta externa como en hospitalización en hospitales periféricos hay mayor cantidad de pacientes con el agravante de no tener tiempo para la academia.

En todos los casos estudiados los médicos consideran que el tiempo del que disponen según su percepción para la realización de la consulta es menor que el que desearían en condiciones ideales Se encontraron diferencias significativas entre el tiempo percibido entre los médicos de los hospitales nacionales y los de los regionales y periféricos, siendo los nacionales siempre los de mejores condiciones. También se encontró influencia de la edad con el tiempo percibido ya que los médicos más jóvenes parecen requerir menos tiempo para realizar sus consultas.

\section{RECOMENDACIONES}

Se recomienda que se establezca una diferencia entre el tiempo de consulta que se asigne a los pacientes por primera vez y de consulta subsecuente en la consulta externa. En consulta por primera vez se recomienda aumentar el tiempo a $40 \mathrm{minu}$ tos por paciente y en la consulta subsecuente a 20 minutos por paciente para disminuir la brecha en los tiempos deseados y asignados. Además de restringir la posibilidad de citas de recargo para garantizar el tiempo en los pacientes con cita programada. El tiempo de realización de interconsultas no debe ser menor a 30 minutos por cada una al igual para la atención de cada paciente hospitalizado.

Se sugiere la asignación uniforme de tiempo dedicado a asuntos académicas en todos los tipos de hospital para que haya igualdad de oportunidades de formación. La cantidad de camas asignadas a cada médico se debería estandarizar y evaluar la recarga de trabajo de los médicos internistas en los hospitales periféricos de la CCSS en este rubro. En todos los hospitales se recomienda la revisión de la cantidad de pacientes tanto de consulta externa como de hospitalización según el tipo de hospital para dar una mejor atención.

\section{BIBLIOGRAFÍA}

1. Caja Costarricense del Seguro Social. Manual Descriptivo de Puestos San Jose, Costa Rica.

2. Colegio de Médicos y Cirujanos de Costa Rica . Acta de Junta de Gobierno Sesion del 26 de junio 2013,San José, Colegio de Médicos y Cirujanos de Costa Rica.

3. Consejo General de los Colegios Oficiales de Médicos de España. Tiempo de trabajo, consulta de la Comisión Europea a los interlocutores sociales. Europa al Día, 2010; 1-6.

4. Cranston, Mark et al. The practice of internal medicine in Europe: organisation, clinical conditions and procedures. EuropeanJournal of Internal Medicine, 2013; 627-632.

5. Departamento de empleo y asuntos sociales. Cuadros de mando de indicadores de calidad en el ámbito de los servicios sociales. País Vasco: Centro de documentación y estudios SIIS. 2011.

6. El Presidente de la República y el Ministro de Salubridad Pública. Reglamento General de Hospitales Nacionales, Ley de la Republica San Jose, Costa Rica

7. Impact of Attending Physician Workload on Patient Care: A Survey of Hospitalists 2013: 375-377

8. Izabella Rohlfs P. Salud, estilos de vida y condiciones de trabajo de los médicos y médicas de Cataluña. Cataluña: Fundación Galatea. 2007.

9. MINGA Consultora S.A. Manual de Análisis de la Dotación de Personal en Establecimientos de Salud. Santiago. OPS. 2004.

10. Social, M. d. (s.f.). Ministerio de Trabajo y Seguridad Social. Recuperado el 6 de Diciembre de 2013, de http://www.mtss.go.cr

11. ¿Qué es Medicina Interna? Revista Médica de Chile2006;(134): 1338-1344

12. Tziner, Ahazron et al. Work stress and turnover intentions among hospital physicians: The mediating role of burnout and work satisfaction. Journal of Work and Organizational Psychology. (2015) Recuperado el 6 de diciembre de 2013 de http//dx.doi./1016/j.rpto.2015.05.001. 


\section{CONFLICTO DE INTERÉS Y/O AGRADE- CIMIENTOS}

Los autores declaran que no existió ningún conflicto de interés en la presente investigación.

\section{FUENTES DE APOYO}

No hay fuentes de apoyo que declarar. 